\title{
Drugs for hypercholesterolaemia - from statins to pro- protein convertase subtilisin kexin 9 (PCSK9) inhibition
}

\author{
Authors: Anthony S Wierzbicki ${ }^{A}$ and Paul Grant ${ }^{B}$
}

Cardiovascular disease (CVD) remains one of the commonest sources of morbidity and mortality in the world. Lipids and especially low density lipoprotein cholesterol (LDL-C) contribute to the risk of CVD events. Statins are the primary therapy for hypercholesterolaemia and recent evidence supports the use of ezetimibe as a second-line agent. Pro-protein convertase subtilisin kexin 9 (PCSK9) is a regulator of LDL receptor expression. Activating mutations in PCSK9 give rise to a form of familial hypercholesterolaemia, while inactivating mutations lead to lower LDL-C levels and fewer CVD events. Therapies to inhibit PCSK9 are in development and two antibody-based therapies - alirocumab and evolocumab - have recently been licensed. This article reviews the actions of PCSK9, the novel therapeutics targeted on this molecule and how they are likely to be used in clinical practice until large scale CVD outcome studies with PCSK9 inhibitors are published.

KEYWORDS: Cardiovascular disease, hyperlipidaemia, PSCK9 inhibitors, statins

\section{Introduction}

Cardiovascular disease (CVD) remains the commonest agerelated manifestation of disease in humans. ${ }^{1,2}$ In the UK, 35\% of individuals will die of CVD and about $50 \%$ will experience a CVD event of some form during their lifetime. It has even been found in ancient Egyptian pharoahs. ${ }^{3}$ The commonest cause of CVD is atherosclerosis. Atheroma may even be an unfortunate consequence of the extension of human lifespans relative to other primates. $^{1,2}$

Atherosclerosis has multiple manifestations in different vascular beds and is driven by multiple CVD risk factors. It is driven by constitutive and modifiable risk factors. Constitutive risk factors include age and gender, which account for most CVD risk. A small component arises from genetic risk factors, including familial hypercholesterolaemia - the commonest autosomal dominant genetic disorder in humans with a prevalence of 1 in $250 ;{ }^{4}$ modifiable risk factors account for the rest and include smoking, diabetes/obesity, hypertension and

Authors: ${ }^{\text {A }}$ consultant in metabolic medicine/chemical pathology, Guy's and St Thomas' Hospitals, London, UK; ' Consultant physician, OCDEM, Churchill Hospital, Headington, Oxford, UK hyperlipidaemia as well as psychosocial factors. ${ }^{5}$ Intervention on smoking remains the most significant risk modifier but is often the least discussed in the medical literature. Most discussions concentrate on other pharmacologically modifiable risk factors, such as lipids, blood pressure and diabetes. Effective treatments exist in all of these fields. Most of the major agents are off-patent and, therefore, cheap. For many years, treatment focused on haemostatic risk factors and blood pressure because they were amenable to successful intervention but over the last 20 years, the availability of statins has enabled the focus to switch to intervening on lipids and especially low density lipoprotein cholesterol (LDL-C). ${ }^{6}$ The frequency of disease and low costs of treatment mean intervention is costeffective for health systems even at low risk thresholds. ${ }^{7,8}$

\section{Novel interventions in cardiovascular disease}

The proponents of the lipid-centric view state that 'no atherosclerosis can happen without LDL-C'. However, CVD events do occur at low concentrations of LDL-C, though this is uncommon. ${ }^{6}$ These cases are likely driven by other components that drive atherosclerosis, including inflammation, immunological activation, blood pressure and diabetes. ${ }^{9}$ Recent studies have advanced the use of non-lipid lowering therapies in preventing CVD. The systolic blood pressure intervention trial (SPRINT) has shown benefits not just on CVD events but also on CVD mortality with lower blood pressures irrespective of the agent used. ${ }^{10}$ The disadvantages were an increase in acute kidney injury and in hypotensive episodes. The likely outcome for guideline purposes will be to increase the number of anti-hypertensive drugs prescribed from two to three and possibly advise doctors to treat all stroke patients with anti-hypertensives, irrespective of initial blood pressure. ${ }^{7}$ In the field of diabetes, the published results of the sodium-glucose-lithium transporter 2 (SGLT-2) inhibitor empagliflozin (empagliflozin cardiovascular outcome event trial in type 2 diabetes mellitus patients, EMPA-REGS) $)^{11}$ and the results of the glucagon-like peptide-1 (GLP-1) agonist liraglutide (liraglutide effect and action in diabetes: evaluation of cardiovascular outcome results, LEADER-6) ${ }^{12}$ trials show reduced mortality (SGLT-2) and CVD events and will lead to the order of different classes of hypoglycaemic therapy in the pathway being rearranged.

\section{Statins and cardiovascular disease}

Statins remain the cornerstone of intervention on lipids given their unprecedented evidence base. ${ }^{6}$ They have a generally 
good adverse effect profile but are associated with problems in some individuals. ${ }^{13}$ The commonest issue, affecting up to $20 \%$ in spontaneous reporting studies and $1-3 \%$ of individuals in well conducted surveys, is myalgia (muscle pain). ${ }^{14}$ The QSTATIN analysis of all statin-related adverse effects, based on a UK primary care sample of 2.2 million patients initiated on statin therapy, identified 1,209 cases of documented myopathy with a number needed to harm of 97 (74-112) in men and 259 (186-375) in women. ${ }^{15}$ Statin-induced myopathy has recently been shown to represent a direct form of mitochondrial myotoxicity. ${ }^{16}$ Other lipid-lowering therapies are less efficacious (eg fibrates ${ }^{17}$ or ezetimibe ${ }^{18}$ ) and have worse adverse effect profiles (bile acid sequestrants). It is in this context that novel lipid-lowering therapies have to be considered. ${ }^{19}$

\section{Pro-protein convertase subtilisin kexin 9}

Pro-protein subtilisin kexin 9 (PCSK9) is a member of a family of molecules that regulate receptors and secreted proteins through proteolysis. ${ }^{20}$ It was originally discovered when a form of familial hypercholesterolaemia was identified in which LDL receptor function was normal in explanted cells but inhibited in the presence of plasma. ${ }^{21}$ The cause was found to be activating mutations in PCSK9 that followed an autosomal dominant pattern of inheritance. Most cases are heterozygotes $^{22}$ though some homozygotes have now been described. ${ }^{23}$ Later studies found that inactivating mutations were associated with $0.4-0.8 \mathrm{mmol} / \mathrm{L}(15-28 \%)$ lower plasma LDL-C concentrations and large reductions in coronary (54-88\%) but not stroke events over 15 years in the Atherosclerosis Risk In Communities (ARIC) Study. ${ }^{24}$ PCSK9 causes internalisation and increased degradation of LDL receptors leading to raised plasma LDL-C concentrations. ${ }^{25}$ An alternative pathway regulating LDL receptor expression, acting through the inducible degrader of LDL receptor (IDOL), also exists but its significance is unclear. ${ }^{26}$ The normal function of PCSK9 seems to be to switch the liver out of the circulation post-prandially, leading to temporary loss of first pass clearance of lipoproteins, thus enabling triglycerides and cholesterol uptake in peripheral tissues. ${ }^{25}$ It seems to be involved in the lipid changes related to adrenarche and the menopause $^{27}$ and in the genesis of the metabolic syndrome ${ }^{28}$ but many details of its control and significance remain to be elucidated. Though most PCSK9 activity is found in the liver, the gene is also expressed in the gut ${ }^{29}$ and in the brain, especially in the cerebellum. ${ }^{30}$ Its function in those tissues is unclear.

\section{Intervention on PCSK9}

The scientific background on the effects of both activating and inactivating mutations of PCSK9, allied with the normal phenotype of homozygous deficient individuals has encouraged the development of pharmacological methods of inhibiting PCSK9. ${ }^{19,31}$ These have ranged from attempts to make small molecule inhibitors of the proteolytic domain of PCSK9 (as it exists in plasma in the form of mutually activated dimers) to inactivating antibodies, anti-sense oligonucleotides and includes attempts at immunisation. The poor bioavailability of small molecule inhibitors has so far limited development. However, there has been more success with reducing PCSK9 levels in plasma either by messenger RNA knockdown by antisense oligonucleotides $^{32}$ or, more commonly, through the use of antibody injections.

\section{Antibody-based therapies}

While numerous companies have developed PCSK9 inhibitors, long-term development has only occurred with antibody-based therapies to date: alirocumab, evolucumab and bococizumab. ${ }^{31}$ All of these are human or humanised antibodies that do not penetrate the blood-brain barrier and are injected fortnightly or monthly. The standard doses are $150 \mathrm{mg}$ alirocumab or $140 \mathrm{mg}$ evolucumab. A lower dose $75 \mathrm{mg}$ option exists for alirocumab. All of these drugs have followed similar phase III trial programmes in patients with established CVD, highrisk primary prevention (including some patients with type 2 diabetes $^{33}$ ), heterozygous familial hypercholesterolaemia ${ }^{34,35}$ and statin-intolerant patients. ${ }^{36,37}$ These analyses are based on an average of 6 months treatment though data exists on up to 2 years of drug exposure in selected populations. PCSK9 inhibitors show consistent effects with average LDL-C reductions of $57 \%$ with $75 / 150 \mathrm{mg}$ alirocumab or $140 \mathrm{mg}$ evolucumab given fortnightly. ${ }^{38}$ Triglycerides were reduced by $15 \%$ and HDL-C rose by $7 \%$. The trials also suggest that PCSK9 inhibition may reduce lipoprotein (a) levels by $24-30 \%{ }^{38,39}$ but has no effect on C-reactive protein levels. ${ }^{40}$ Tolerability is generally good with injection site reactions in $1-2 \%$ of patients and no obviously distinct adverse effect profile apart maybe from a small increase in influenza and nasopharyngitis, ${ }^{41}$ although $2-8 \%$ of patients do discontinue treatment in these studies. Antibodies are made to anti-PCSK9 antibody therapies but occur at low frequency and seem to have no effect in reducing the efficacy of the drugs.

One surprise from the programmes was the efficacy of PCSK9 inhibitors in patients with homozygous familial hypercholesterolaemia. A study with evolucumab showed variation in response ranging from nil in LDL receptor deficient (null) to up to $45 \%$ LDL-C reduction in LDL receptor defective (point mutation) patients. ${ }^{42}$ This implies that a trial of PCSK9 inhibition is worthwhile in all patients being considered for apheresis because while both copies of LDL-receptor in these patients contain mutations, they may still have some residual activity (except if the LDL receptor has been truncated because of a frame shift or deletion mutation).

\section{Use in statin-intolerant populations}

Statin intolerance has been defined in consensus statements as intolerance to three drugs tried at different doses. ${ }^{43,44}$ Formal re-challenge is necessary to confirm the diagnosis given the frequency of non-specific muscle aches in the population. The trials of PCSK9 inhibitors have included studies in statinintolerant populations. ${ }^{36,45}$ The ODYSSEY alternative study randomised 361 patients with a history of muscle-based intolerance to two or more statins to non-statin methods of LDL-C reduction (alirocumab or ezetimibe) as well as rechallenge with atorvastatin $20 \mathrm{mg}$ or placebo. ${ }^{36}$ The study had a placebo run-in phase. The efficacy of PCSK9 intervention on LDL-C was similar to that in statin-tolerant populations with reductions in LDL-C of $45 \%$ for $75 / 150 \mathrm{mg}$ alirocumab, $32 \%$ for $20 \mathrm{mg}$ atorvastatin and $15 \%$ for ezetimibe. In the study, $13 \%$ discontinued during the placebo run-in phase with 
$50 \%$ stating the cause was due to muscle related side effects (19\% myalgia and 15\% muscle spasms). During the 24-week blinded therapy phase, 25-35\% discontinued treatment - again mainly due to myalgia. Rates of discontinuation were $18 \%$ for alirocumab and $25 \%$ for either ezetimibe or atorvastatin therapy (relative risk $0.61(0.38-0.99) ; \mathrm{p}=0.04)$. However, once in the open label phase of study, $90 \%$ persisted with statin therapy if previously assigned to that group. In the GAUSS-2 (goal achievement after utilising an anti-PCSK9 antibody in statin-intolerant subjects) trial, 307 patients were randomised to different evolocumab regimens, placebo or ezetimibe for 12 weeks. In the evolucumab arms, $12 \%$ had adverse events and LDL-C was reduced by $53-55 \%$ as opposed to $23 \%$ adverse events and a $16 \%$ LDL-C reduction with ezetimibe. ${ }^{37}$ In the GAUSS- 3 trial with evolucumab in 491 patients, $43 \%$ had muscle symptoms with $20 \mathrm{mg}$ atorvastatin but not while taking a placebo in phase $A$. In phase B, 218 patients were randomised 2:1 to evolocumab or ezetimibe therapy. Ezetimibe reduced LDL-C by $17 \%$, while evolocumab reduced LDL-C by $55 \%$. Muscle symptoms were reported by $29 \%$ of patients on ezetimibe, of whom $7 \%$ discontinued, and $21 \%$ on evolucumab with $1 \%$ discontinuing. ${ }^{46}$ Thus, much myalgia associated with statins is highly likely to represent pre-existing osteological or neurological complaints and not be related to treatment with these agents.

\section{Outcome of phase III studies}

The completion of the phase III programmes for both alirocumab and evolucumab allows general conclusions to be drawn in meta-analyses. ${ }^{38,41,47}$ These analyses are based on an average of 2 years of drug exposure in selected populations in trials with multiple exclusion criteria. Although the lipid efficacy data is likely sound, the adverse effect data has to be considered preliminary and any changes in CVD events - a $50 \%(95 \% \mathrm{CI},-10 \%$ to $77 \%)$ reduction and a reduction in allcause mortality of $55 \%$ ( $95 \%$ CI, $14 \%$ to $77 \%)$ - speculative as they are based on low numbers of events. ${ }^{48,49}$ Meta-analyses of the registration trials have been performed and show reductions in LDL-C from $47-53 \%$ (low dose alirocumab) to $54-58 \%$ (top dose alirocumab or evolocumab) for these agents, with good tolerability even in statin-intolerant patients. The studies identify an increase in neurocognitive events (relative risk 1.54; 19 versus 5 events after correction for multiple publication) as a potential effect of PCSK9 inhibitors ${ }^{47}$ but the symptoms are non-specific, numbers very low and likely mechanisms obscure. The only known scientific fact is that PCSK9 is expressed in the cerebellum but its function there is unknown. ${ }^{30}$ Neurological substudies within CVD outcomes trials may identify whether these effects are significant. ${ }^{50}$

\section{Limitations of phase III trials with PCSK9 inhibitors}

It is likely that only exposure to PCSK9 therapy of other clinical groups not recruited to the phase III trials will expose any limitations of these agents. Phase III studies did not identify either myalgia (as opposed to myositis/ rhabdomyolysis) or diabetes as adverse effects of statins. These emerged in clinical practice - in the case of statin-induced hyperglycaemia, increasing transitions to a diagnosis of type 2 diabetes, after 15 years of general use. ${ }^{51}$ No trial data have yet been published on the efficacy and safety of PCSK9 inhibitors in patients with renal dyslipidaemia, liver disease, HIV infection or significant hypertriglyceridaemia $(>4.5 \mathrm{mmol} / \mathrm{L})$ or in children and adolescents. Some of these studies are now underway. A concern not addressed in trials to date may be the use of PCSK9 inhibitors in patients with hepatitis virus infection - especially hepatitis $\mathrm{C}$ - as these viruses exploit the LDL-receptor to enter and exit cells. ${ }^{52}$ PCSK9 knockout mice show increased rates of pancreatic abnormalities, beta-cell function and hepatic steatosis, ${ }^{53}$ although these changes have not been seen in humans apart from one individual report of pancreatitis. $^{45}$

\section{The current context of LDL-C reduction therapies}

The introduction of PSCK9 inhibitors comes at a time when the treatment of lipid-related risk factors for CVD is evolving. The improved reduction of outcomes: vytorin efficacy international trial (IMPROVE-IT) has shown an incremental 7\% benefit of ezetimibe added to optimised baseline statin therapy in patients with acute coronary syndromes but only for non-fatal CVD events $(32.7 \%$ versus $34.7 \%$ CVD events at 7 years; $p=0.02){ }^{18}$ The greatest effects were seen in patients with type 2 diabetes or older patients, ie those at highest absolute risk. In high-risk patients, it will likely reset treatment paradigms either to lower LDL-C targets (approximately $1.4 \mathrm{mmol} / \mathrm{L}$ ) or, more likely, to the addition of ezetimibe to treatment regimens in patients with the highest absolute CVD risk.

In 2016, PCSK9 inhibitors will join the formulary for lipid intervention in many countries. The National Institute for Health and Care Excellence has recently reviewed both evolocumab ${ }^{54}$ and alirocumab ${ }^{55}$ for common indications in the context of current guidelines on lipid management. ${ }^{56}$ Homozygous familial hypercholesterolaemia was outside the scope of the review though PCSK9 inhibitors are likely to be accepted for use in that indication. Based on their efficacy and safety data, the provisional recommendations for the use of these drugs within the NHS are outlines in Table $1 .{ }^{54,55}$

CVD outcome studies with PCSK9 inhibitors are underway (ODYSSEY $^{57}$ and FOURIER ${ }^{58}$ - further cardiovascular outcomes research with PCSK9 inhibition in subjects with elevated risk study) and will determine their utility in general CVD risk populations. These studies were designed and recruited for before the evidence became available for ezetimibe and thus their baseline recruitment LDL-C for trial was in the region 2.0-2.5 mmol/L ( $2.34 \mathrm{mmol} / \mathrm{L}$ in FOURIER $\left.{ }^{58}\right)$. Whatever the results of these studies, it is likely that PCSK9 drugs will be thirdline agents after ezetimibe. ${ }^{59}$ Their CVD benefits (if confirmed) may be marginal in the general population and only significant in terms of numbers needed to treat (or in health economic terms) in patient groups with high LDL-C (statin intolerant patients or those with familial hypercholesterolaemia). ${ }^{54,55,60}$ Their high cost relative to other lipid-lowering agents may limit their use and many other alternative approaches to modifying lipid levels are in development. ${ }^{19}$

\section{Conclusion}

PCSK9 inhibitors are novel therapeutic interventions that reduce LDL-C by $54-58 \%$ with low rates of injection site reactions and discontinuation. They are a useful addition to the 


\begin{tabular}{|c|c|c|c|}
\hline & \multirow[t]{2}{*}{ Without CVD } & \multicolumn{2}{|c|}{ With CVD } \\
\hline & & High risk of CVD* & Very high risk of CVD** \\
\hline $\begin{array}{l}\text { Primary non-familial hypercholesterolaemia } \\
\text { or mixed dyslipidaemia }\end{array}$ & $\begin{array}{l}\text { Not recommended at any } \\
\text { LDL-C concentration }\end{array}$ & $\begin{array}{l}\text { Recommended only if } \\
\text { persistent LDL-C }>4.0 \mathrm{mmol} / \mathrm{L}\end{array}$ & $\begin{array}{l}\text { Recommended only if } \\
\text { persistent LDL-C }>3.5 \mathrm{mmol} / \mathrm{L}\end{array}$ \\
\hline $\begin{array}{l}\text { Primary heterozygous-familial } \\
\text { hypercholesterolaemia }\end{array}$ & $\begin{array}{l}\text { Recommended only if } \\
\text { persistent } \mathrm{LDL}-\mathrm{C}>5.0 \mathrm{mmol} / \mathrm{L}\end{array}$ & $\begin{array}{l}\text { Recommended only if } \\
\text { persistent } L D L-C>3.5 \mathrm{mmol} / \mathrm{L}\end{array}$ & $\begin{array}{l}\text { Recommended only if } \\
\text { persistent } L D L-C>3.5 \mathrm{mmol} / \mathrm{L}\end{array}$ \\
\hline
\end{tabular}

armamentarium of lipid-lowering drugs. For the next few years, until outcomes evidence becomes available and data on adverse effects from registries accumulate, it will be up to individual clinicians reviewing individual patients to decide what the strategy for initiating PCSK9 therapy should be but, to date, data suggest that they will be useful in a subgroup of high-risk patients with high residual LDL-C.

\section{Conflicts of interest}

Professor Wierzbicki chaired the NICE lipid modification guideline CG181and was a clinical expert for the NICE technology appraisals of evolucumab (ID765) and alirocumab (ID779). The views expressed in this article are his own and do not represent those of NICE.

\section{References}

1 Wick G, Berger P, Jansen-Durr P, Grubeck-Loebenstein B. A Darwinian-evolutionary concept of age-related diseases. Exp Gerontol 2003;38:13-25.

2 Wick G, Jakic B, Buszko $M$ et al. The role of heat shock proteins in atherosclerosis. Nat Rev Cardiol 2014;11:516-29.

3 Thompson RC, Allam AH, Lombardi GP et al. Atherosclerosis across 4000 years of human history: the Horus study of four ancient populations. Lancet 2013;381:1211-22.

4 Gidding SS, Ann Champagne M, de Ferranti SD et al. The agenda for familial hypercholesterolemia: a scientific statement from the American Heart Association. Circulation 2015;132:2167-92.

5 Yusuf S, Hawken S, Ounpuu S et al. Effect of potentially modifiable risk factors associated with myocardial infarction in 52 countries (the INTERHEART study): case-control study. Lancet 2004;364:937-52.

6 Baigent C, Blackwell L, Emberson J et al. Efficacy and safety of more intensive lowering of LDL cholesterol: a meta-analysis of data from 170,000 participants in 26 randomised trials. Lancet 2010;376:1670-81.

7 Thomopoulos C, Parati G, Zanchetti A. Effects of blood pressure lowering on outcome incidence in hypertension: 7. Effects of more vs. less intensive blood pressure lowering and different achieved blood pressure levels - updated overview and meta-analyses of randomized trials. J Hypertens 2016;34:613-22.

8 Mihaylova B, Emberson J, Blackwell L et al. The effects of lowering LDL cholesterol with statin therapy in people at low risk of vascular disease: meta-analysis of individual data from 27 randomised trials. Lancet 2012;380:581-90.

9 Grundtman C, Wick G. The autoimmune concept of atherosclerosis. Curr Opin Lipidol 2011;22:327-34.

10 SPRINT Research Group, Wright JT Jr., Williamson JD et al. A randomized trial of intensive versus standard blood-pressure control. N Engl J Med 2015;373:2103-16.
11 Zinman B, Wanner C, Lachin JM et al. Empagliflozin, cardiovascular outcomes, and mortality in type 2 diabetes. $N$ Engl J Med 2015;373:2117-28.

12 Marso SP, Daniels GH, Brown-Frandsen K et al. Liraglutide and cardiovascular outcomes in type 2 diabetes. N Engl J Med 2016; DOI: 10.1056/NEJMoa1603827.

13 Naci H, Brugts J, Ades T. Comparative tolerability and harms of individual statins: a study-level network meta-analysis of 246955 participants from 135 randomized, controlled trials. Circ Cardiovasc Qual Outcomes 2013;6:390-9.

14 Zhang H, Plutzky J, Skentzos S et al. Discontinuation of statins in routine care settings: a cohort study. Ann Intern Med 2013;158:526-34.

15 Hippisley-Cox J, Coupland C. Unintended effects of statins in men and women in England and Wales: population based cohort study using the QResearch database. BMJ 2010;340:c2197.

16 Schirris TJ, Renkema GH, Ritschel T et al. Statin-induced myopathy is associated with mitochondrial complex III inhibition. Cell Metab 2015;22:399-407.

17 Jun M, Foote C, Lv J et al. Effects of fibrates on cardiovascular outcomes: a systematic review and meta-analysis. Lancet 2010;375:1875-84.

18 Cannon CP, Blazing MA, Giugliano RP et al. Ezetimibe Added to Statin Therapy after Acute Coronary Syndromes. N Engl J Med 2015;372:2387-97.

19 Hajhosseiny R, Sabir I, Khavandi K, Wierzbicki AS. The ebbs and flows in the development of cholesterol-lowering drugs: prospects for the future. Clin Pharmacol Ther 2014;96:64-73.

20 Seidah NG. What lies ahead for the proprotein convertases? Ann N Y Acad Sci 2011;1220:149-61.

21 Abifadel M, Varret M, Rabes JP et al. Mutations in PCSK9 cause autosomal dominant hypercholesterolemia. Nat Genet 2003;34:154-6.

22 Hopkins PN, Defesche J, Fouchier SW et al. Characterization of autosomal dominant hypercholesterolemia caused by PCSK9 gain of function mutations and its specific treatment with alirocumab, a PCSK9 monoclonal antibody. Circ Cardiovasc Genet 2015;8:823-31.

23 Alves AC, Etxebarria A, Medeiros AM et al. Characterization of the first PCSK9 gain of function homozygote. J Am Coll Cardiol 2015;66:2152-4.

24 Cohen JC, Boerwinkle E, Mosley TH Jr., Hobbs HH. Sequence variations in PCSK9, low LDL, and protection against coronary heart disease. N Engl J Med 2006;354:1264-72.

25 Horton JD, Cohen JC, Hobbs HH. PCSK9: a convertase that coordinates LDL catabolism. J Lipid Res 2009;50 Suppl:S172-7.

26 Sasaki M, Terao Y, Ayaori M et al. Hepatic overexpression of idol increases circulating protein convertase subtilisin/kexin type 9 in mice and hamsters via dual mechanisms: sterol regulatory elementbinding protein 2 and low-density lipoprotein receptor-dependent pathways. Arterioscler Thromb Vasc Biol 2014;34:1171-8.

27 Lakoski SG, Lagace TA, Cohen JC et al. Genetic and metabolic determinants of plasma PCSK9 levels. J Clin Endocrinol Metab 2009;94:2537-43. 
28 Costet P, Cariou B, Lambert G et al. Hepatic PCSK9 expression is regulated by nutritional status via insulin and sterol regulatory element-binding protein 1c. J Biol Chem 2006;281:6211-8.

29 Levy E, Ben Djoudi Ouadda A, Spahis S et al. PCSK9 plays a significant role in cholesterol homeostasis and lipid transport in intestinal epithelial cells. Atherosclerosis 2013;227:297-306.

30 Rousselet E, Marcinkiewicz J, Kriz J et al. PCSK9 reduces the protein levels of the LDL receptor in mouse brain during development and after ischemic stroke. J Lipid Res 2011;52:1383-91.

31 Wierzbicki AS, Hardman TC, Viljoen A. Inhibition of pro-protein convertase subtilisin kexin 9 (PCSK9) as a treatment for hyperlipidaemia. Expert Opin Investig Drugs 2012;21:667-76.

32 Fitzgerald K, Frank-Kamenetsky M, Shulga-Morskaya S et al. Effect of an RNA interference drug on the synthesis of proprotein convertase subtilisin/kexin type 9 (PCSK9) and the concentration of serum LDL cholesterol in healthy volunteers: a randomised, single-blind, placebo-controlled, phase 1 trial. Lancet 2014;383:60-8.

33 Sattar N, Preiss D, Robinson JG et al. Lipid-lowering efficacy of the PCSK9 inhibitor evolocumab (AMG 145) in patients with type 2 diabetes: a meta-analysis of individual patient data. Lancet Diabetes Endocrinol 2016;4:403-10.

34 Kastelein JJ, Ginsberg HN, Langslet G et al. ODYSSEY FH I and FH II: 78 week results with alirocumab treatment in 735 patients with heterozygous familial hypercholesterolaemia. Eur Heart $J$ 2015;36:2996-3003.

35 Raal FJ, Stein EA, Dufour R et al. PCSK9 inhibition with evolocumab (AMG 145) in heterozygous familial hypercholesterolaemia (RUTHERFORD-2): a randomised, double-blind, placebo-controlled trial. Lancet 2015;385:331-40.

36 Moriarty PM, Thompson PD, Cannon CP et al. Efficacy and safety of alirocumab vs ezetimibe in statin-intolerant patients, with a statin rechallenge arm: The ODYSSEY ALTERNATIVE randomized trial J Clin Lipidol 2015;9:758-69.

37 Stroes E, Colquhoun D, Sullivan D et al. Anti-PCSK9 antibody effectively lowers cholesterol in patients with statin intolerance: the GAUSS-2 randomized, placebo-controlled phase 3 clinical trial of evolocumab. J Am Coll Cardiol 2014;63:2541-8.

38 Lipinski MJ, Benedetto U, Escarcega RO et al. The impact of proprotein convertase subtilisin-kexin type 9 serine protease inhibitors on lipid levels and outcomes in patients with primary hypercholesterolaemia: a network meta-analysis. Eur Heart $J$ 2016;37:536-45.

39 Raal FJ, Giugliano RP, Sabatine MS et al. Reduction in lipoprotein(a) with PCSK9 monoclonal antibody evolocumab (AMG 145): a pooled analysis of more than 1,300 patients in 4 phase II trials. J Am Coll Cardiol 2014;63:1278-88.

40 Sahebkar A, Di Giosia P, Stamerra CA et al. Effect of monoclonal antibodies to PCSK9 on high-sensitivity C-reactive protein levels: a meta-analysis of 16 randomized controlled treatment arms. $\mathrm{Br} \mathrm{J}$ Clin Pharmacol 2016; 81:1175-90.

41 Zhang XL, Zhu QQ, Zhu L et al. Safety and efficacy of anti-PCSK9 antibodies: a meta-analysis of 25 randomized, controlled trials. BMC Med 2015;13:123.

42 Raal FJ, Honarpour N, Blom DJ et al. Inhibition of PCSK9 with evolocumab in homozygous familial hypercholesterolaemia (TESLA Part B): a randomised, double-blind, placebo-controlled trial. Lancet 2015;385:341-50.

43 Stroes ES, Thompson PD, Corsini A et al. Statin-associated muscle symptoms: impact on statin therapy-European Atherosclerosis Society Consensus Panel Statement on Assessment, Aetiology and Management. Eur Heart J 2015;36:1012-22.
44 Mancini GB, Tashakkor AY, Baker S et al. Diagnosis, prevention, and management of statin adverse effects and intolerance: Canadian Working Group Consensus update. Can J Cardiol 2013;29:1553-68.

45 Sullivan D, Olsson AG, Scott R et al. Effect of a monoclonal antibody to PCSK9 on low-density lipoprotein cholesterol levels in statin-intolerant patients: the GAUSS randomized trial. JAMA 2012;308:2497-506.

46 Nissen SE, Stroes E, Dent-Acosta RE et al. Efficacy and tolerability of evolocumab vs ezetimibe in patients with muscle-related statin intolerance: the GAUSS-3 randomized clinical trial. JAMA 2016;315:1580-90.

47 Kolodziejczak M, Navarese EP. Role of PCSK9 antibodies in cardiovascular disease: Critical considerations of mortality and neurocognitive findings from the current literature. Atherosclerosis 2016;247:189-92.

48 Sabatine MS, Giugliano RP, Wiviott SD et al. Efficacy and safety of evolocumab in reducing lipids and cardiovascular events. $N$ Engl $J$ Med 2015;372:1500-9.

49 Robinson JG, Farnier M, Krempf M et al. Efficacy and safety of alirocumab in reducing lipids and cardiovascular events. $N$ Engl J Med 2015;372:1489-99.

50 Evaluating PCSK9 binding antibody influence on cognitive health in high cardiovascular risk subjects (EBBINGHAUS). Registered 31 July 2014. Available online at https://clinicaltrials.gov/ct2/show/ NCT02207634 [Accessed 9 May 2016].

51 Sattar N, Preiss D, Murray HM et al. Statins and risk of incident diabetes: a collaborative meta-analysis of randomised statin trials. Lancet 2010;375:735-42.

52 Syed GH, Tang H, Khan M et al. Hepatitis C virus stimulates lowdensity lipoprotein receptor expression to facilitate viral propagation. J Virol 2014;88:2519-29.

53 Mbikay M, Sirois F, Mayne J et al. PCSK9-deficient mice exhibit impaired glucose tolerance and pancreatic islet abnormalities. FEBS Lett 2010;584:701-6.

54 National Institute for Health and Care Excellence. Evolocumab for treating primary hypercholesterolaemia and mixed dyslipidaemia. NICE technology appraisal guideline ID765. London: NICE, 2015.

55 National Institute for Health and Care Excellence. Alirocumab for treating primary hypercholesterolaemia and mixed dyslipidaemia. NICE technology appraisal guideline ID779. London: NICE, 2015.

56 Rabar S, Harker M, O'Flynn N et al. Lipid modification and cardiovascular risk assessment for the primary and secondary prevention of cardiovascular disease: summary of updated NICE guidance. BMJ 2014;349:g4356.

57 Schwartz GG, Bessac L, Berdan LG et al. Effect of alirocumab, a monoclonal antibody to PCSK9, on long-term cardiovascular outcomes following acute coronary syndromes: rationale and design of the ODYSSEY outcomes trial. Am Heart J 2014;168:682-9.

58 Sabatine MS, Giugliano RP, Keech A et al. Rationale and design of the further cardiovascular outcomes research with PCSK9 inhibition in subjects with elevated risk trial. Am Heart J 2016;173:94-101.

59 National Institute for Health and Care Excellence. Ezetimibe for treating primary heterozygous-familial and non-familial hypercholesterolaemia. NICE technology appraisal guidance TA385. London: NICE, 2016.

60 Weintraub WS, Gidding SS. PCSK9 inhibitors: a technology worth paying for? Pharmacoeconomics 2016;34:217-20.

Address for correspondence: Prof A S Wierzbicki, Department of Chemical Pathology, St. Thomas' Hospital, Lambeth Palace Road, London SE1 7EH, UK.

Email: Anthony.wierzbicki@kcl.ac.uk 